\title{
A Modular Model of Reversible Heat Pumps and Chillers for System Applications
}

\author{
Fabian Wüllhorst $^{1} \quad$ David Jansen $^{1} \quad$ Philipp Mehrfeld $^{1} \quad$ Dirk Müller $^{1}$ \\ ${ }^{1}$ Institute for Energy Efficient Buildings and Indoor Climate, E.ON Energy Research Center, RWTH Aachen \\ University, Germany, fabian.wuellhorsteeonerc.rwth-aachen.de
}

\begin{abstract}
Vapour compression machines such as heat pumps and chillers are vital for achieving climate goals. Efficiency of both depend mostly on system integration. In order to simulate coupled energy systems, fast and stable simulation models are required. Hence, we implement an open-source model for reversible vapour compression machines. The black-box based refrigeration cycle is replaceable, additional inertia and losses are optional. Furthermore, we model relevant safety controls of vapour compression machines. To show validity of the presented approach, we first calibrate two different black-box models onto measured heat pump data. The table-based model fits both measured temperature and power with minimal calibration effort. Second, we show influences of different model options onto coupled building performance simulations. Computation time increases up to $50 \%$ when enabling all model options. Simultaneously, seasonal efficiency decreases by up to $23 \%$ when modeling all safety controls. Keywords: Heat Pump, Chiller, Modular Model
\end{abstract}

\section{Introduction}

Heating and cooling account for half of Europe's secondary energy demand (Heat Roadmap Europe 2017). While cooling is already mainly achieved using electrically driven chillers (e.g. air conditioners), heat pumps (HPs) are set to replace gas or oil fired boilers (IEA 2020). Thus, improving efficiency of both is a major goal of current research.

As both chiller and heat pump are based on the same vapour compression cycles, designation mainly depends on which side contains the usable heat flow. Using a fourway reversing valve, a heat pump may act as a chiller and vice versa. Hence, in the following we refer to both as vapour compression machines (VCM).

To increase efficiency of VCMs, improvements on component level and system integration level are possible. Generally, the efficiency depends on the applied temperature levels. These temperature levels on the other hand depend mainly on the system integration. Thus, we focus on the integration of VCM into an energy system.

To thoroughly assess efficiency on system level, seasonal coefficients of performance (SCOPs) are required (Huchtemann and Dirk Müller 2012). While
Hardware-in-the-Loop experiments as in Mehrfeld, Nürenberg, Knorr, et al. (2020) or field tests are cost intensive, model-based simulation analysis offers a viable solution to not only assess the SCOP but also to find promising control and system designs that optimize efficiency.

A model is always created based on the aim of the simulation analysis (VDI 3633:2014-12 2014). Consequently, numerous approaches for the modeling of VCMs exist in literature. The following section will give a brief overview.

\section{Related work}

The main distinction in models is between two types: Modeling the refrigeration cycle with empirical data (black-box) and using physical-based equation (gray/white-box) (Jin 2002). Black-box models are usually robust and require less computation time compared to the latter. In most cases, empirical data is based on steady-state operating points. Outside the given performance maps, which are often small, extrapolations usually yield non-physical results (Cimmino and Wetter 2017). However, for certain investigation aims, partly higher accuracies are achieved compared to gray-box models (Carbonell Sánchez et al. 2012). Afjei and Dott (2011) assign investigation targets to the different model types. Graybox models are, due to the high level of detail and the high flexibility, suitable for the investigation of new heat pump concepts. For dynamic simulations on the other hand, black-box models with dynamic effects are useful (Afjei and Dott 2011).

\subsection{Gray-box models}

Cimmino and Wetter (2017) introduce two gray-box models, scrollwaterTowater and ReciprocatingWaterToWater. By assuming a simplified refrigeration circuit, the modeling of the expansion valve is not required. Both models are nonreversible. As refrigerant, R410A is used. HP internal safety control is taken into account using temperature protection. By calibrating the model with manufacturer data, the parameters are defined and made accessible via records (Cimmino and Wetter 2017). Dechesne et al. (2017) model an inverter-controlled air/water HP with consideration of the refrigeration circuit for the refrigerant R410A. The possible icing of the evaporator 
is taken into account through various assumptions. However, a validation of these assumptions is not performed (Dechesne et al. 2017). The model of a heat pump with water as refrigerant is implemented by Chamoun, Rulliere, Haberschill, and Berail (2012) for industrial applications. In another contribution, they introduce a screw compressor model for HP applications (Chamoun, Rulliere, Haberschill, and Peureux 2013).

\subsection{Black-box models}

In the following, two black-box models of the IBPSA library will be described (International Building Performance Simulation Association 2018). In each, the two variables electricity consumption $P_{\mathrm{el}}$ and used heat flow $\dot{Q}_{\text {Con }}$ are determined. Assuming a steady-state, adiabatic process, $\dot{Q}_{\mathrm{Eva}}$ is then calculated:

$$
0=P_{\mathrm{el}}+\dot{Q}_{\mathrm{Eva}}-\dot{Q}_{\mathrm{Con}}
$$

The models Carnot_TCon and Carnot_y are based on the same principle, only the input variable changes. Carnot_TCon sets the output temperature of the condenser $T_{\text {Con.out }}$ via an ideal heater. Using the output $\dot{Q}_{\text {Con }}$ and the COP, $P_{\mathrm{el}}$ and $\dot{Q}_{\mathrm{Eva}}$ are calculated. Carnot_y determines $P_{\mathrm{el}}$ by the product of $P_{\mathrm{el}, \mathrm{Nom}}$ and the input $y$ which is the part load set point between zero and one. For both models, the COP is obtained using the Carnot efficiency at nominal and current conditions. These model approaches are valid for both chillers and heat pumps. For chillers, condenser and evaporator are switched (International Building Performance Simulation Association 2018).

For heat pumps, the AixLib library introduced an approach obtaining $P_{\mathrm{el}}$ and $\dot{Q}_{\mathrm{Con}}$ through manufacturer data based on EN 14511 (EN 14511-1:2018-03 2018; D. Müller et al. 2016). Based on the same approach, the IDEAS library contains a heat pump model as well (Jorissen et al. 2018).

Besides these open-source models, researchers have presented their approach in various contributions. De Coninck et al. (2010) calculates $P_{\mathrm{el}}$ and $\dot{Q}_{\mathrm{Con}}$ based on the ambient temperature $T_{\mathrm{amb}}$, the condenser temperature $T_{\text {Con }}$, and manufacturer data. Wystrcil and Kalz (2012) use field test data to determine a four coefficient polynomial of the $C O P$ as a function of the temperatures $T_{\text {Eva,in }}$ and $T_{\text {Con,out }}$.

Until now, we focused our review on Modelica models. However, the modeling approaches in other simulation tools mostly do not differ. The assumption in Equation 1 is state of research for black-box approaches. Examples are given in (Afjei and Wetter 1997; EN 12900:2013-10 2013).

This review highlights the gap in the current state of the art, which can be summarized in two points.

1. Each contribution develops a new model. However, the black-box approaches only differ in how $P_{\mathrm{el}}$ and
$\dot{Q}_{\text {Use }}$ are calculated. To spend less time in model development and more time in simulation analysis, a uniform and modular approach is necessary.

2. Besides Cimmino and Wetter (2017), VCM internal safety controls are disregarded. However, such controls strongly influence the behaviour of the machine in the energy system and thus, it's SCOP (Mehrfeld, Nürenberg, Knorr, et al. 2020).

Following the identified gaps, we present a new modular model for reversible heat pumps and chillers. The vapour compression cycle is modeled as a black-box. Contrary, the interactions with the energy systems are gray-box based. The model is presented in the following section.

\section{Modular modeling approach}

The following section presents our modeling approach. The overall model is depicted in Figure 1. To ensure reuseability of the approach, we implement all presented models in the open-source library AixLib (D. Müller et al. 2016). We aggregate all relevant inputs and outputs using a pre-defined expandable bus connector. All bus variables are listed in Table 1. In general, set indicates a control variable and Mea a physical variable. As core of each VCM, we start with the black-box approach of the vapour compression cycle.

Table 1. Pre-defined variables of the VCM bus.

\begin{tabular}{ll}
\hline Variable & Explanation \\
\hline nSet & Relative compressor frequency \\
modeSet & Set to false to reverse the device \\
onOffMea & Boolean indicating if device is on \\
PelMea & Measured electrical power \\
CoPMea & Measured COP \\
iceFacMea & Icing factor $f_{\text {ice }}$, see subsection 3.1 \\
TOdaMea & Outdoor air temperature \\
TEvaInMea & Evaporator inlet temperature \\
TEvaOutMea & Evaporator outlet temperature \\
TEvaAmbMea & Ambient temperature at evaporator \\
TConInMea & Condenser inlet temperature \\
TConOutMea & Condenser outlet temperature \\
TConAmbMea & Ambient temperature at condenser \\
\hline
\end{tabular}

\subsection{Vapour compression cycle}

In order to realize a reversible and modular VCM model, we introduce a partial model named PartialPerformanceData. Inputs to the model are all bus variables listed in Table 1. Outputs are the three main energy flows, $\dot{Q}_{\text {Con,out }}, \dot{Q}_{\text {Eva,out }}$ and $P_{\text {el,out }}$. Both HP and chiller models may extend this model and connect these inputs to the outputs based on a black-box approach.

The vapour compression cycle model, named innercycle, then uses this partial model as a re- 


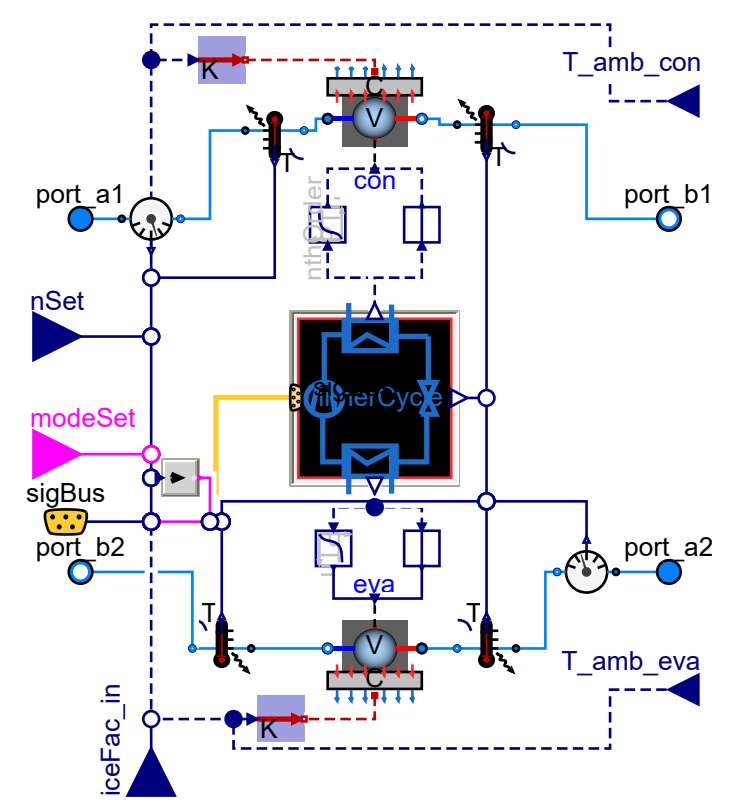

Figure 1. Partial model of a reversible thermal machine

placeable option. Based on the Boolean input modeset, the cycle switches between data for cooling and heating. This approach is visualized in Figure 2. At the

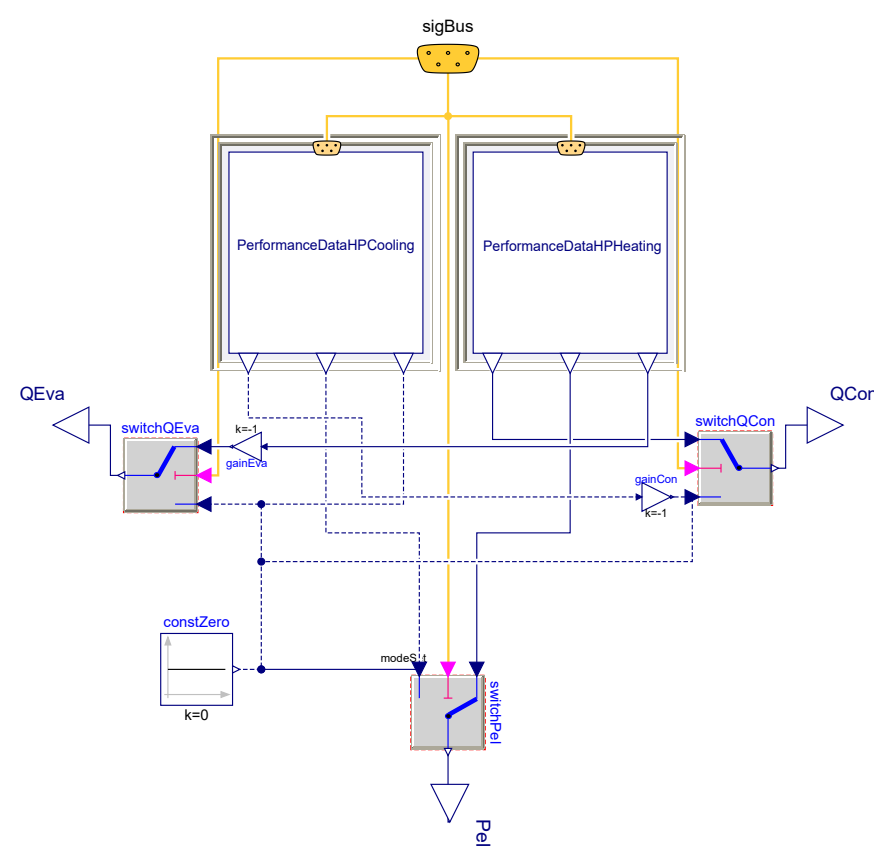

Figure 2. Black-box model of the reversible vapour compression cycle, exemplified for a HP

current state, we have introduced three performance data approaches.

1. Functional approach: Any modelica function using variables in Table 1 to calculate the outputs.

2. 2D-Data: All VCMs on the market require data according to the EN 14511 (EN 14511-1:2018-03 2018). As table inputs, $T_{\mathrm{Eva}, \text { in }}$ and $T_{\mathrm{Con} \text {,out }}$ are re- garded. For non fixed-speed devices, the compressor speed is necessary to model the part load behaviour. We account for part load by linearly multiplying model outputs with nset. Note however, that table data was not necessarily obtained at full load. Hence, we introduce a third approach.

3. 3D-Data: Also based on EN 14511-1:2018-03 (2018), we add compressor speed as a table dependency. While this solves the part load issue, only some manufacturer publish tables this detailed.

All the above-mentioned approaches include two further options. First, a scaling factor may be used to scale the VCM according to demand. Second, we model the VCM internal effect of possible evaporator frosting. As we chose a black-box approach for the vapour compression cycle, gray-box modeling of frost is challenging. Hence, we adapt a black-box approach as well. The icing factor $f_{\text {ice }}$ accounts for losses in evaporator efficiency due to frosting effects. This factor has to be calculated using external models, e.g. according to the COP correction proposed by Afjei and Wetter (1997). For output of the inner cycle it follows:

$$
\begin{aligned}
\dot{Q}_{\text {Eva }, \text { out }} & =f_{\text {ice }} \cdot \dot{Q}_{\mathrm{Eva}} \\
P_{\text {el,out }} & =P_{\mathrm{el}} \\
\dot{Q}_{\text {Con }, \text { out }} & =\dot{Q}_{\text {Eva,out }}+P_{\text {el,out }}
\end{aligned}
$$

\subsection{Inertia}

As stated in the prior section, the innercycle model is based on stationary data points. Hence, transient calculation of the performance in the vapour compression cycle does not take place. This is especially problematic during on-off switching of the device. When the compressor is switched on, it requires instantaneous electrical power. However, the refrigerant and components have mass and thermal inertias. As a result, the effective heat flow is delayed. Thus, we consider this inertia of the inner cycle by adding a CriticalDamping filter. This adds an PT delay of n-th order on the black-box outputs $\dot{Q}_{\mathrm{Con}}$ and $\dot{Q}_{\mathrm{Eva}}$. Again, usage and order of the filter are custom parameters.

\subsection{Heat exchangers}

The heat flow rates calculated in the Innercyle are directly fed into the secondary fluids. Current open-source models neglect heat losses with the ambient. However, the dynamics during heat up and cool down phases may be relevant for the system interaction. Thus, we extend the heat exchanger HeaterCooler_u from the IBPSA. We add a capacity between secondary fluid and ambient air, refer to Figure 3. Thus, the ambient temperature influences the secondary fluids. Heating the device up takes longer due to higher capacity. Similarly, the capacity may transfer heat to the secondary fluid during cool down, even though the device is turned off. As some black-box data implicitly accounts for such losses, not all use cases require this option. It's usage is thus optional. 


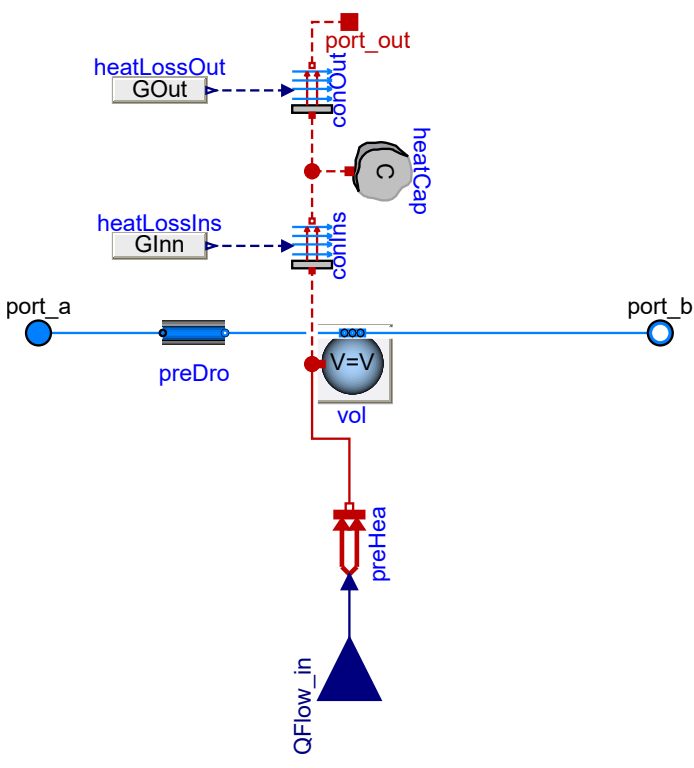

Figure 3. Model for evaporator and condenser

\subsection{Safety controls}

In section 2 we point out that current model approaches neglect interaction of system controls with the internal safety control of VCMs. As only the interaction with the system is relevant to us, we regard all safety controls which influence the long term performance of the system level as relevant. Furthermore, the order of safety controls is relevant in Modelica. We choose the following order, going from set point generators over logical controls to physical safety controls.

- Thermal disinfection: Frequent thermal disinfection of domestic hot water is required for HPs to ensure safety of users. Temperatures over $60^{\circ} \mathrm{C}$ are necessary (Van Kenhove 2018).

- Defrost: For air-source VCMs, evaporators may need a defrosting control to ensure an operation without frost on the coils. For reversible VCMs, we model reverse cycle defrost. If the VCM is not reversible, defrost is modeled using auxiliary heaters.

- On/off control: Most VCMs require a minimal run time, a minimal off-time and forbid frequent on/off switching. All three requirements depend on nset and pre (nSet). Thus, they are included in one model. Switching single requirements on or off is achieved through booleans.

- Operational envelope: The compressor of VCMs can only operate in a given operational envelope. If outside of the envelope, pressure switches and thermal resistors shut the VCM off. In the model, we select TConOutMea and TEvaInMea as input for the model. As in Cimmino and Wetter (2017), a hysteresis prevents frequent switching.
- Frost protection: For water or brine sources, the fluid temperature may not fall below certain thresholds to prevent phase change. Hence, we add a safety control to ensure TEvaOutMea and TConInMea are greater than a given threshold.

All controls aggregated, the resulting SafetyController is depicted in Figure 4. Each option may be disabled. To better analyze simulation results, integer outputs show users the number of times the control was used.

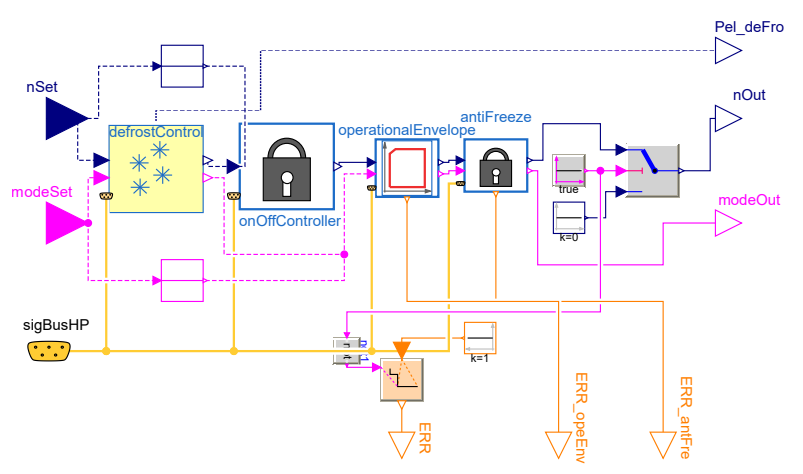

Figure 4. Implemented safety controller for the VCM

\subsection{System integration}

Lastly, we adapt a model of the overall VCM system by adding additional components used in VCM. These include secondary pumps, fans and an auxiliary heater. Again, all listed components are replaceable and optional. Not optional is the system controller. It includes the safety controls and the control to generate the signals nSet and modeset.

This system model is currently implemented for a heat pump. An example is located in the package AixLib. Systems. HeatPumpSystems.

For reference, the models may be viewed and tested on the latest commit 38c19751b in the repository AixLib ${ }^{1}$.

\section{Heat pump calibration}

To show validity and modularity of our presented approach, we calibrate a brine/water HP with different blackbox approaches. As calibration method, we select the approach presented in Mehrfeld, Nürenberg, and Dirk Müller (2021).

Experiments are performed at a Hardware-in-the-Loop test bench. Temperature inputs, outputs and consumed $\hat{P}_{\mathrm{e}}$ are presented in Figure 5. Measured data is denoted using the hat operator, e.g. $\hat{T}$. The data between 6000 and $8000 \mathrm{~s}$ data is not used for calibration but for validation only. We calibrate the NRMSE, refer to Equation 5, of both $P_{\mathrm{el}}$ and $T_{\text {Con,out }}$ weighted equally.

$$
N R M S E=\frac{1}{\hat{x}_{\max }-\hat{x}_{\min }} \cdot \sqrt{\frac{\sum_{i=1}^{n}\left(x_{i}-\hat{x}_{i}\right)^{2}}{n}}
$$

\footnotetext{
${ }^{1}$ https://github.com/RWTH-EBC/AixLib
} 
Table 2. Data of the HP on the test bench according to EN 14511

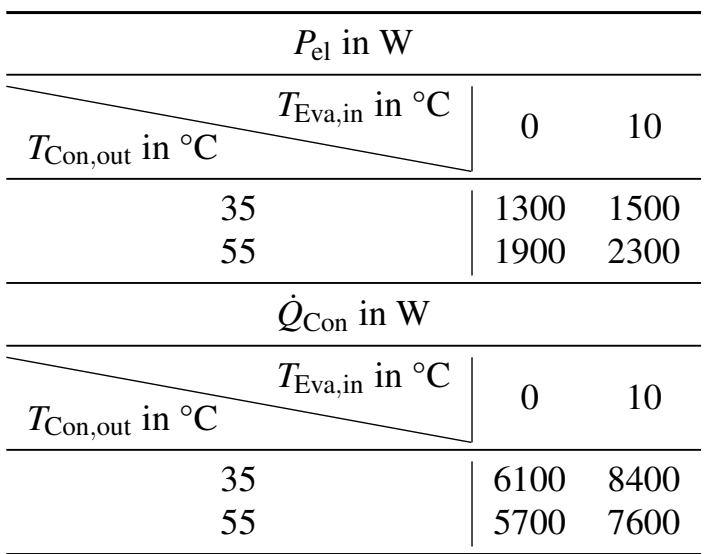

As calibration parameters, we select only those which are most sensitive to our target values. These parameters are mass flow rate at condenser $\dot{m}_{\text {Con }}$, volume of condenser $V_{\text {Con }}$ and frequency of the inertia filter $f_{\text {inertia. }}$. First, we

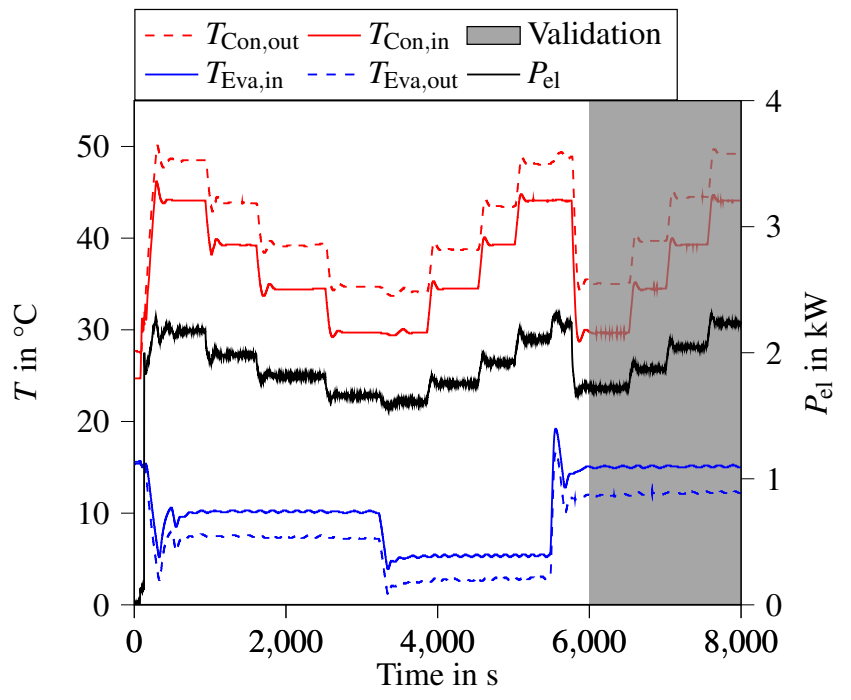

Figure 5. Experimental data for the calibration and validation

model the HP according to manufacturer data listed in standard EN 14511 (EN 14511-1:2018-03 2018). The data is stated in Table 2. Second, we use a Carnot approach with a constant quality grade. The consumed electrical power is constant at nominal conditions. This approach is equivalant to the Carnot approach in the IBP SA for on/off heat pumps. The equations are as follows:

$$
\begin{aligned}
P_{\mathrm{el}} & =P_{\mathrm{el}, \mathrm{Nom}} \\
\dot{Q}_{\mathrm{Con}} & =P_{\mathrm{el}, \mathrm{Nom}} \cdot \xi \cdot \frac{T_{\mathrm{Con}, \mathrm{out}}}{T_{\mathrm{Con}, \mathrm{out}}-T_{\mathrm{Eva}, \text { in }}}
\end{aligned}
$$

In this study, the quality grade $\xi$ and nominal power $P_{\mathrm{el}, \text { Nom }}$ are additional calibration parameters.

\subsection{Results and Discussion}

The resulting parameter values of both calibrations are listed in Table 3 together with applied boundaries. Ad- ditionally, time series plots for both cases are displayed in Figure 6.

Table 3. Applied calibration parameters and results

\begin{tabular}{lcccc}
\hline Parameter & Min & Max & \multicolumn{2}{c}{ Results } \\
& & & Table & Carnot \\
\hline$\dot{m}_{\text {Con }}$ in $\mathrm{kg} \mathrm{s}^{-1}$ & 0.2 & 0.6 & 0.404 & 0.214 \\
$V_{\mathrm{Con}}$ in $\mathrm{L}$ & 1 & 50 & 4.47 & 1.59 \\
$f_{\text {inertia }}$ in $\mu \mathrm{Hz}$ & 1 & 300 & 12 & 13.2 \\
$\xi$ in $\%$ & 5 & 50 & - & 43.18 \\
$P_{\text {el,Nom }}$ in $\mathrm{kW}$ & 1.5 & 2.5 & - & 1.88 \\
\hline \hline NRMSE Calibration & & 0.046 & 0.053 \\
NRMSE Validation & & 0.041 & 0.055 \\
\hline
\end{tabular}

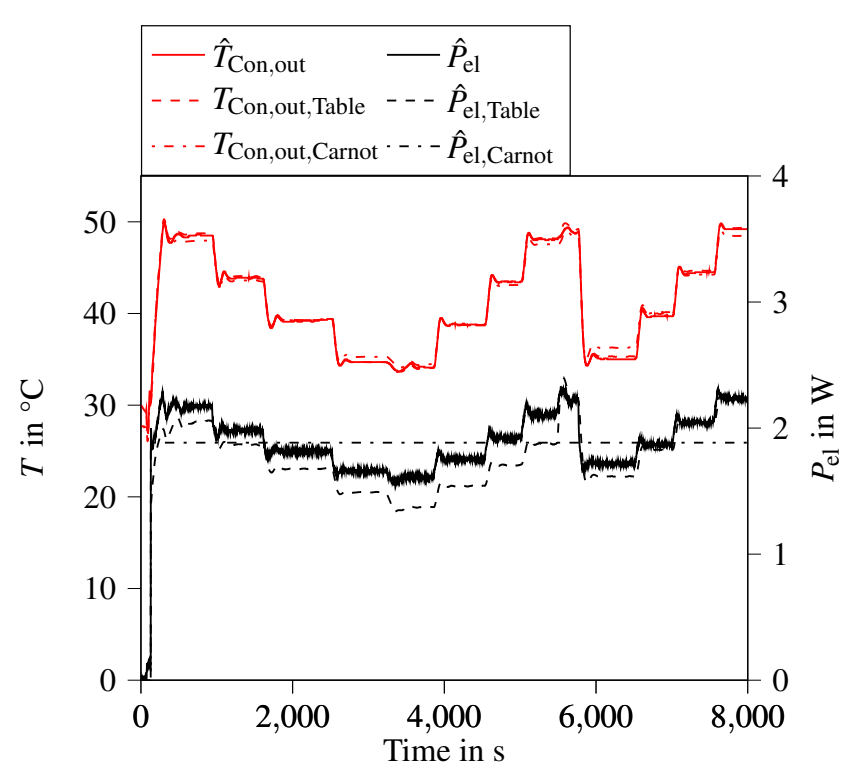

Figure 6. Time series plots for objectives of calibration.

First of all, both model approaches are able to reproduce the measured temperature. This is possible due to the dynamics introduced by the additional inertia and volume of the heat exchanger. For the validation part, source temperature increases and thus induces an extrapolation of the black-box approach. Hence, the error increases slightly.

However, the black-box is based on just four points (Table) or two parameters (Carnot). As interpolation typically yields sufficient accuracy, both approaches may be used for typical operation limits for brine/water HPs. Looking at the electrical power, both approaches differ. Obviously, the constant $P_{\mathrm{el}, \mathrm{Nom}}$ leads to error prone model outputs. Not as obvious, the measured data according to EN 14511-1:2018-03 (2018) also shows discrepancies. Most interestingly, the error is smaller during validation. As $T_{\text {Con,out,Table }}$ is higher than $\hat{T}_{\text {Con,out }}$ during validation, simulated $P_{\text {el.Table }}$ increases as well. Note that the results of the calibration depend on the weighting of objectives. With a higher weighting towards $P_{\mathrm{el}}$, the fit would increase. 
Concluding, both approaches may be used based on the aim of the simulation analysis. Minimal data input of just four data points or two calibrated parameters yield accurate representations when compared to experimental data.

\section{Seasonal building performance sim- ulations}

The main goal of our contribution is to develop a modular model for the use in energy systems. Hence, our second use case concerns the integration of said model into a building energy system.

The building is based on the high-order approach in the AixLib (D. Müller et al. 2016). Demand of domestic hot water is neglected.

An air/water HP is simulated, hence the anti-freeze protection function is not applied. The simulation period is equal to one heating period, which is defined from $1^{\text {st }}$ October to the $30^{\text {th }}$ April. Besides computation time and the number of state events, the SCOP is calculated.

Model parameters are selected based on calibration results, scaled to the manufacturer data of an exemplary fixed-speed HP. Thermal disinfection is performed once a week by increasing the storage tank temperature above $60^{\circ} \mathrm{C}$ for a duration of $15 \mathrm{~min}$. If the outdoor temperature falls below $5^{\circ} \mathrm{C}$, defrost cycles are modeled by a daily 10 min reversal of the refrigeration circuit.

To demonstrate influences of introduced models, we analyze eight model configurations (MC). Together with relevant results, they are listed in Table 4. The simulation settings are stated in Table 5

Table 4. Definition and results of model configurations. Usage of option is indicated by (x).

\begin{tabular}{|c|c|c|c|c|c|c|c|c|c|}
\hline 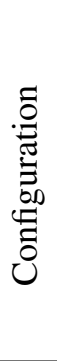 & 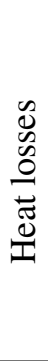 & 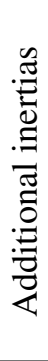 & 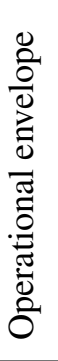 & 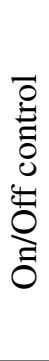 & 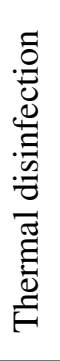 & $\begin{array}{l}\vec{\infty} \\
\stackrel{0}{0} \\
\stackrel{0}{0}\end{array}$ & 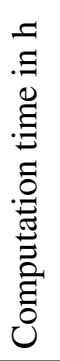 & 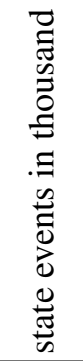 & 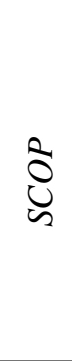 \\
\hline 1 & o & o & 0 & 0 & o & $\mathrm{O}$ & 5.2 & 46.1 & 2.44 \\
\hline 2 & $\mathrm{X}$ & $\mathrm{x}$ & o & $\mathrm{O}$ & o & o & 6.9 & 45.7 & 2.21 \\
\hline 3 & $\mathrm{X}$ & o & $\mathrm{x}$ & $\mathrm{X}$ & o & $\mathrm{O}$ & 6.5 & 64.8 & 2.14 \\
\hline 4 & o & $\mathrm{x}$ & $\mathrm{X}$ & $\mathrm{X}$ & o & o & 7.2 & 61.8 & 2.36 \\
\hline 5 & $\mathrm{X}$ & $\mathrm{x}$ & $\mathrm{X}$ & $\mathrm{X}$ & o & o & 7.4 & 64.7 & 2.15 \\
\hline 6 & $\mathrm{X}$ & $\mathrm{X}$ & $\mathrm{X}$ & $\mathrm{X}$ & $\mathrm{X}$ & o & 7.5 & 65.3 & 2.14 \\
\hline 7 & $\mathrm{X}$ & $\mathrm{x}$ & $\mathrm{x}$ & $\mathrm{X}$ & o & $\mathrm{X}$ & 7.8 & 65.6 & 1.88 \\
\hline 8 & $\mathrm{X}$ & $\mathrm{X}$ & $\mathrm{X}$ & $\mathrm{X}$ & $\mathrm{X}$ & $\mathrm{X}$ & 7.8 & 66.0 & 1.88 \\
\hline
\end{tabular}

\subsection{Results and Discussion}

Looking at Table 4, we want to analyze two influences in detail.
Table 5. Simulation settings for all studied configurations

\begin{tabular}{lr}
\hline Setting & Value \\
\hline Start time & 273 days \\
Stop time & 485 days \\
Interval length & 5 min \\
Solver & Dassl \\
Tolerance & 0.0001 \\
Store variables at events & true \\
\hline
\end{tabular}

First, the computation time in $\mathrm{MC}_{2}$ increased by a factor of 1.33. As both heat losses and inertias are active in $\mathrm{MC}_{2}$, the direct impact of both options is not distinguishable. By comparing $\mathrm{MC}_{3}$ and $\mathrm{MC}_{4}$ to $\mathrm{MC}_{5}$, the impact can be assessed. Neglecting the system inertias in $\mathrm{MC}_{3}$ leads to a $12.6 \%$ reduction in computation time compared to $\mathrm{MC}_{5}$. If the heat losses are not simulated, the computation time decreases by $3.6 \%$ compared to $\mathrm{MC}_{5}$. Despite increased number of state events, $\mathrm{MC}_{3}$ is faster than $\mathrm{MC}_{2}$. Overall, the CriticalDamping block of third order for mapping the inertia increases the computation time without having a noteworthy impact on the SCOP. This increase may be attributed in six additional continuous time states introduced by the model option. The usage of heat losses only introduce two new continuous time states. In general, the usage of inertias should always be weighted against the increased computation time.

Second, we analyze the influence of safety controls on the computation time and SCOP. Both the computation time and the number of state events increase between $\mathrm{MC}_{1}$ and $\mathrm{MC}_{3}$ to $\mathrm{MC}_{8}$. The compliance with the operating envelope and on/off control evokes 15000-20000 state events depending on the $\mathrm{MC}$, resulting in an increase of the computation time by at least $7.5 \%$. The operating envelope is not exceeded at any time. Therefore, this increase is due to compliance with the switching cycles. In $\mathrm{MC}_{6}$, the influence of thermal disinfection is analyzed. Compared to $\mathrm{MC}_{5}$, the SCOP is decreased by $0.5 \%$, and the computation time is increased by $1.2 \%$. The chosen period of $15 \mathrm{~min}$ as well as the week-based circuit are possible reasons for the small influence. Overall, the defrost control has the greatest influence on the SCOP. As a result of reversing the refrigeration circuit, the SCOP decreases by $12.6 \%$. On the one hand, this is due to the fact that the table data already take defrosting losses into account. On the other hand, the duration and timing of the defrosting cycles in the use case are not based on validated assumptions. Following defrost control, the heat losses show the highest impact on the SCOP. Comparing $\mathrm{MC}_{1}$ to $\mathrm{MC}_{2}$, the SCOP decreases by $9.4 \%$. To show that this decrease is not induced by the additional inertias, we compare $\mathrm{MC}_{4}$ to $\mathrm{MC}_{5}$. Here, the decrease amounts for $8.9 \%$. This is explained by the fact that inertias change the dynamics of heat flow and not the quantity itself. 
Generally, the obtained SCOP may seem small. However, we examined a supply temperature of $55^{\circ} \mathrm{C}$ using a $2 \mathrm{~K}$ bandwidth hysteresis control. For these systems, the SCOPs are in similar ranges compared to SCOPs obtained in field tests (Huchtemann and Dirk Müller 2012).

\section{Conclusion}

A modular model for reversible heat pumps and chillers is presented. The vapour compression cycle is modeled as a replaceable black-box. Contrary, relevant interactions with the whole energy system are modeled as gray-box. Most notably, safety controls such as on/off controls and the operational envelope are regarded in the model. All models are optional, enabling users to choose modeling depth based on their simulation aim.

To demonstrate validity of our approach, we calibrate two different black-box approaches onto measured data. While both manage to reproduce supply temperatures, the Carnot based approach fails to match electricity demands.

Besides calibration, we show influences of different model options onto simulation speed and efficiency of heat pumps. The presented results indicate that safety controls should always be regarded neglected on system integration level.

While the presented approach is reversible, our background focuses mainly on the use of VCM as a heat pump in building energy systems. Calibration and validation of chiller applications may show limitations of the approach. Especially validation of experiments using a four-way reversing valve are of interest. As we neglect internal effects of the VCM, losses or dynamics during reverse cycle mode may lead to poor model accuracy.

Last but not least, we already implemented various black-box approaches in the AixLib (D. Müller et al. 2016). Researchers and practitioners are invited to contribute new black-box approaches to this open-source modeling library. Thus, the variety of simulation aims covered by the presented modeling approach may increase, yielding a more thorough understanding of heat pumps and chillers in system applications.

\section{Acknowledgements}

\section{References}

Afjei, Thomas and Ralf Dott (2011). "Heat pump modelling for annual performance, design and new technologies". In: 12th Conference of International Building Performance Simulation Association, pp. 1-8.

Afjei, Thomas and Michael Wetter (1997). Compressor heat pump including frost and cycle losses. URL: https : / / simulationresearch.lbl.gov/wetter/download/type204_hp. pdf.

Carbonell Sánchez, Daniel et al. (2012). "Numerical analysis of heat pumps models: comparative study between equation-fit and refrigerant cycle based models". In: Solar energy for a brighter future: book of proceedings: EuroSun 2012.
Chamoun, Marwan, Romuald Rulliere, Philippe Haberschill, and Jean Francois Berail (2012). "Dynamic model of an industrial heat pump using water as refrigerant". In: International Journal of Refrigeration 35.4, pp. 1080-1091. ISSN: 0140-7007. DOI: https://doi.org/10.1016/j.ijrefrig.2011.12. 007. URL: https://www.sciencedirect.com/science/article/pii/ S0140700711003082.

Chamoun, Marwan, Romuald Rulliere, Philippe Haberschill, and Jean-Louis Peureux (2013). "Modelica-based modeling and simulation of a twin screw compressor for heat pump applications". In: Applied Thermal Engineering 58.1, pp. 479489. ISSN: 1359-4311. DOI: https : // doi . org/10 . 1016/ j . applthermaleng . 2013 .04 .020. URL: https : / / www . sciencedirect.com/science/article/pii/S1359431113002901.

Cimmino, Massimo and Michael Wetter (2017-07). "Modelling of Heat Pumps with Calibrated Parameters Based on Manufacturer Data". en. In: pp. 219-226. DOI: 10.3384/ ecp17132219. URL: https://ep.liu.se/en/conference-article. aspx ? series $=$ ecp \& issue $=132 \&$ Article_No $=22$ (visited on 2021-04-17).

De Coninck, Roel et al. (2010). "Modelling and simulation of a grid connected photovoltaic heat pump system with thermal energy storage using Modelica”. In: 8th international conference on system simulation, p. 21.

Dechesne, Bertrand et al. (2017). "Comparison of a dynamic model and experimental results of a residential heat pump with vapor injection and variable speed scroll compressor". In: The 30th International Conference on Efficiency, Cost, Optimization, Simulation and Environmental Impact of Energy Systems.

EN 12900:2013-10 (2013-10). Refrigerant compressors - Rating conditions, tolerances and presentation of manufacturer's performance data. Tech. rep.

EN 14511-1:2018-03 (2018-03-14). Air Conditioners, Liquid Chilling Packages and Heat Pumps for Space Heating and Cooling and Process Chillers, with Electrically Driven Compressors - Part 1: Terms and Definitions. Tech. rep. Bruxelles, Belgium: CEN/TC 113. URL: https://standards.cen.eu/ dyn/www/f?p=204:110:0::::FSP_PROJECT:59177\&cs $=$ 18B95E474391A98AD6EAB83913BAE7714.

Heat Roadmap Europe (2017). Heating and Cooling - facts and figures. URL: https://www.isi.fraunhofer.de/content/dam/ isi/dokumente/cce/2017/29882_Brochure_Heating - and Cooling_web.pdf.

Huchtemann, Kristian and Dirk Müller (2012). "Evaluation of a field test with retrofit heat pumps". In: Building and Environment 53, pp. 100-106. ISSN: 03601323. DOI: 10.1016/j. buildenv.2012.01.013.

IEA (2020). Heat Pumps. URL: https://www.iea.org/reports/ heat-pumps.

International Building Performance Simulation Association (2018). IBPSA Project 1: BIM/GIS and Modelica Framework for building and community energy system design and operation. URL: https://ibpsa.github.io/project1/.

Jin, Hui (2002). "Parameter estimation based models of water source heat pumps". Dissertation. Shanghai: Jiaotong University.

Jorissen, Filip et al. (2018). "Implementation and Verification of the IDEAS Building Energy Simulation Library". In: Journal of Building Performance Simulation 11 (6), pp. 669-688. DOI: 10.1080/19401493.2018.1428361.

Mehrfeld, Philipp, Markus Nürenberg, Martin Knorr, et al. (2020). "Dynamic evaluations of heat pump and micro com- 
bined heat and power systems using the hardware-in-theloop approach". In: Journal of Building Engineering 28, p. 101032.

Mehrfeld, Philipp, Markus Nürenberg, and Dirk Müller (2021). "Model Calibration of an Air Source Heat Pump System for Transient Simulations in Modelica". en. In: 13th IEA Heat Pump Conference. Jeju, Korea, p. 11.

Müller, D. et al. (2016). "AixLib - An Open-Source Modelica Library within the IEA-EBC Annex 60 Framework". In: BauSIM 2016.

Van Kenhove, Elisa (2018). "Coupled Thermohydraulic and Biologic Modelling of Legionella Pneumophila Proliferation in Domestic Hot Water Systems". PhD Thesis. Gent: University Gent.

VDI 3633:2014-12 (2014-12). Simulation of systems in materials handling, logistics and production - Fundamentals. Tech. rep.

Wystrcil, Dominik and Doreen Kalz (2012). "Thermohydraulische Modellierung eines Niedrigexergiesystems zur Gebäudeheizung- und kühlung und exergetische Bewertung von Regelungsstrategien”. In: Fourth German-Austrian IBPSA Conference. 